\title{
Molecular Epidemiology of Bordetella Pertussis in Iran from March 2015 to January 2018
}

Samaneh Saedi, Vajihe sadat Nikbin, Masoumeh Nakhost Lotfi, Sedaghatpour Shahab, Fereshteh Shahcheraghi

Microbiology research center, Department of bacteriology Pasteur Institute of Iran

\section{Background:}

Whooping cough is a highly contagious infection of the lungs and airways, is mainly caused by Bordetella pertussis. Right now the highest rates of morbidity and mortality of the disease were reported in infants and other age groups usually display milder symptoms of the disease. Sience 1950s the whole cell pertussis vaccine (WCVs) combined with tetanus and diphtheria toxoids (DTP) was introduced. Nowaday, while a majority of developed countries switched from the use of whole cell Pertussis vaccines (WCV) to acellular (ACV), developing countries continue to use WCV-based combination vaccines. In Iran, pertussis remains an endemic infection and incidence has presented a significant decrease after vaccination since1990s. According to the World Health Organization (WHO) reports, (DTP) vaccine coverage in iran is estimated to be greater than $96 \%$ since 2000 , in last 3 years ago, we experience a descent trend in cases from 115 cases in 2014,145 cases in 2015 and 116 case in 2016.

We aimed to learn more about epidemic isolates and to monitor transmission of pertussis infection used pulsed-field gel electrophoresis (PFGE) typing and sequence of virulence associated proteins, pertussis toxin (PTX) and pertussis toxin promotor $(\mathrm{ptxP})$, that collected in different provinces of Iran from patients and two vaccine strains used in this study were Bp509 and Bp134.

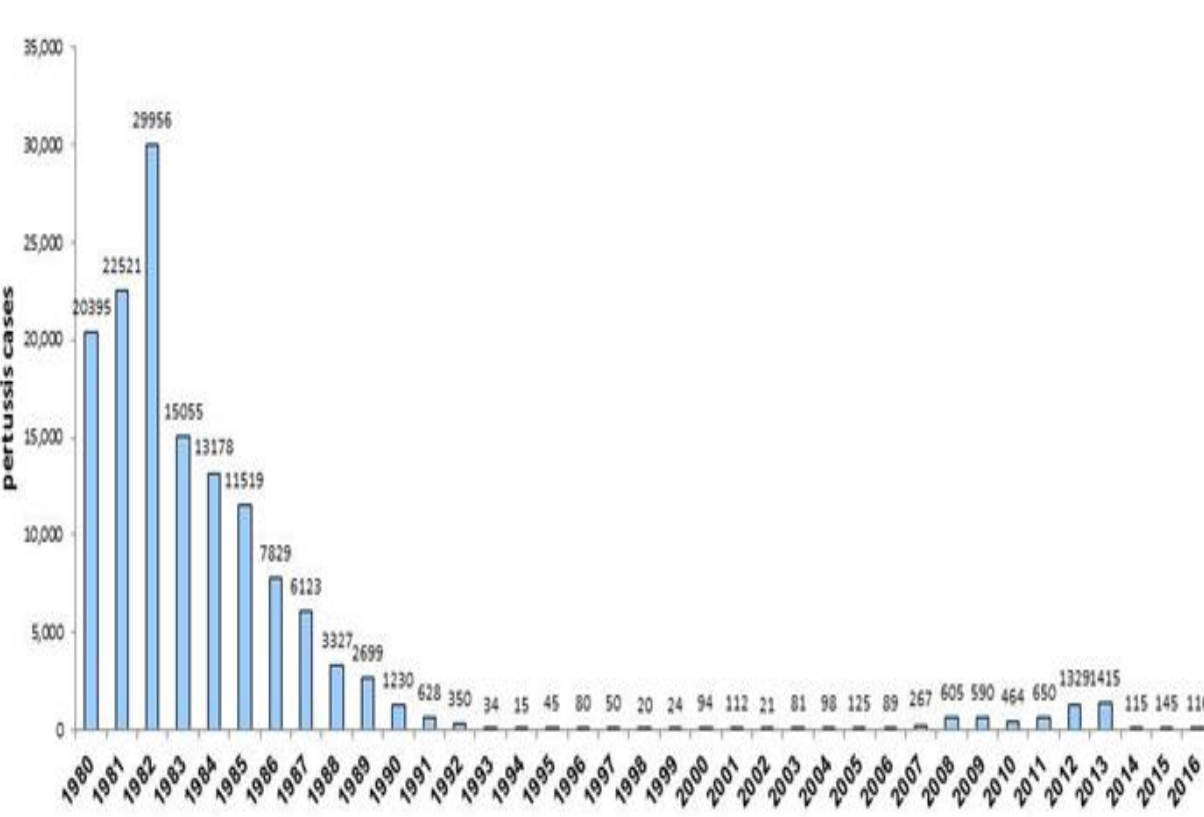

Methods:

We studied 50 B. pertussis isolates collected in different provinces of Iran from patients and send to Pertussis Reference Laboratory, Institute Pasteur of Tehran, Iran between 2015 2018.Tohama I(ATCC BAA-589) as reference strain and two vaccine strains used in this study were Bp509, and Bp134. Information on age, gender and vaccination was available.Pertussis strains were confirmed by biochemical tests and molecular confirmation by specific primers targeting insertion sequence 481 (IS481),100(IS1002). PFGE profiles were determined using a method based on single Xbal digest of the DNA. All samples were selected for sequencing of the polymorphic pertussis toxin promoter (ptxP) and pertussis toxin $\mathrm{S} 1$ ( subunitA). Finally Fragments patterns of PFGE were analyzed with GelCompare II software and dendrogram was drawn by UPGMA.

\section{Results:}

Among our patients, the most affected age group was infants below the age of one, with a notification rate of $70 \%, 20 \%$ were in the age group $1-5$ years and $10 \%$ of cases were aged 10 years. In this study we used Xbal enzyme to classify the vaccine strains and local isolates into PFGE profile groups. A total of 16 distinct PFGE profiles were identify. We found four strains with the PFGE genomic pattern similar to vaccine strain $B$. pertussis 134 with the $\mathrm{ptxP}_{1}$ virulence profile. We have major group of isolates had comprised 22 isolates and it is our dominan circulating strain with representative $\mathrm{ptxP}_{3}$ and $\operatorname{ptxA}_{1}$

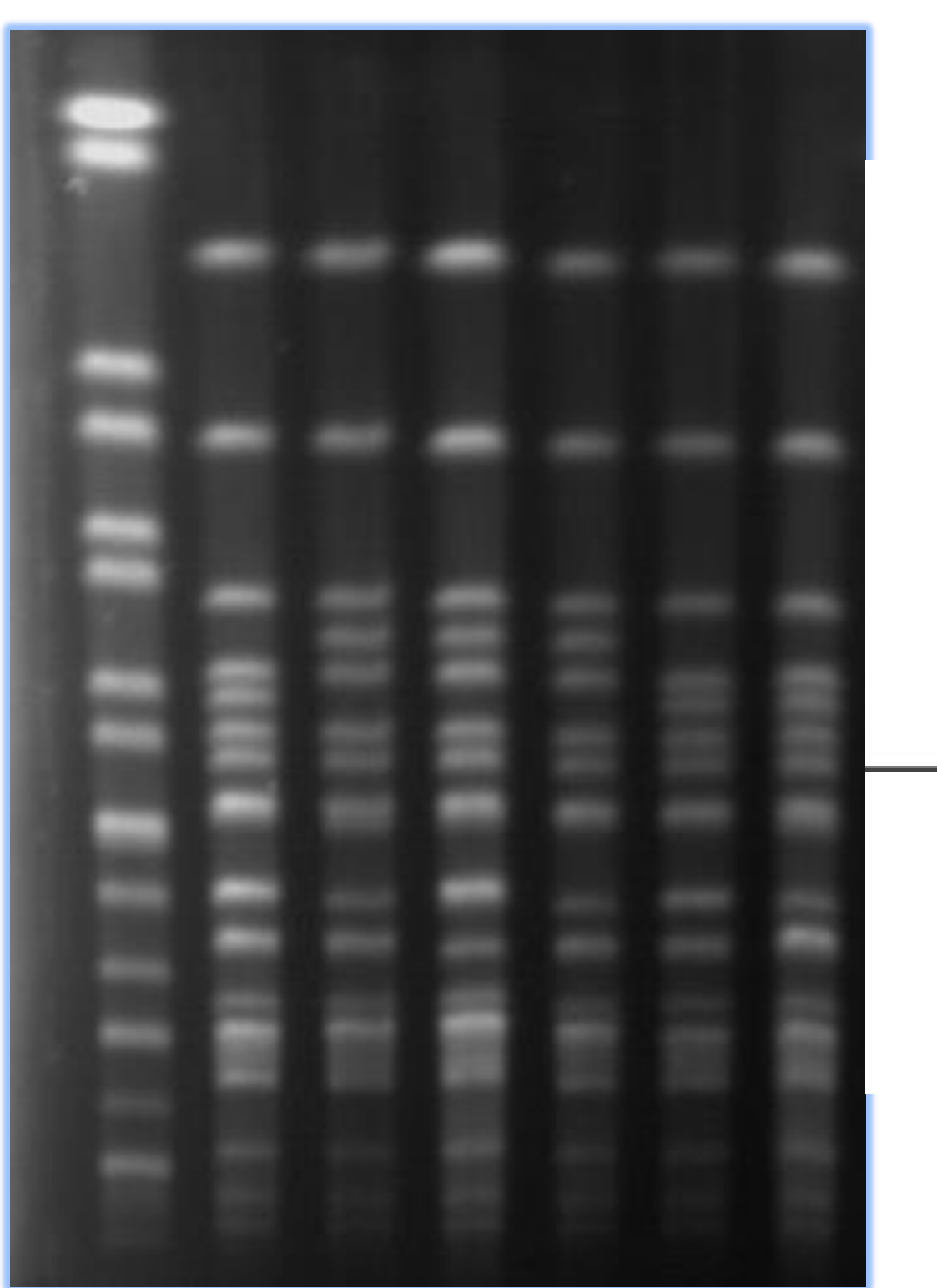

Conclusions:

Remarkable changes in B. pertussis populations have been observed after the introduction of vaccinations. The main reason is the role for pathogen adaptation in the resurgence of pertussis. These changes include antigenic divergence with vaccine strains and increased production of pertussis toxin. Strain variation has progressed in iran, so that we observed 16 distinct PFGE profile among 50 iranian bordetella pertussis isolates. in recent years emerged with a ptxP3 strains have risen to predominance in iran. We found that our dominant cluster like Swedish dominant cluster(BpSR11) with $\operatorname{ptxA} A_{1}, \operatorname{ptxP}_{3}$
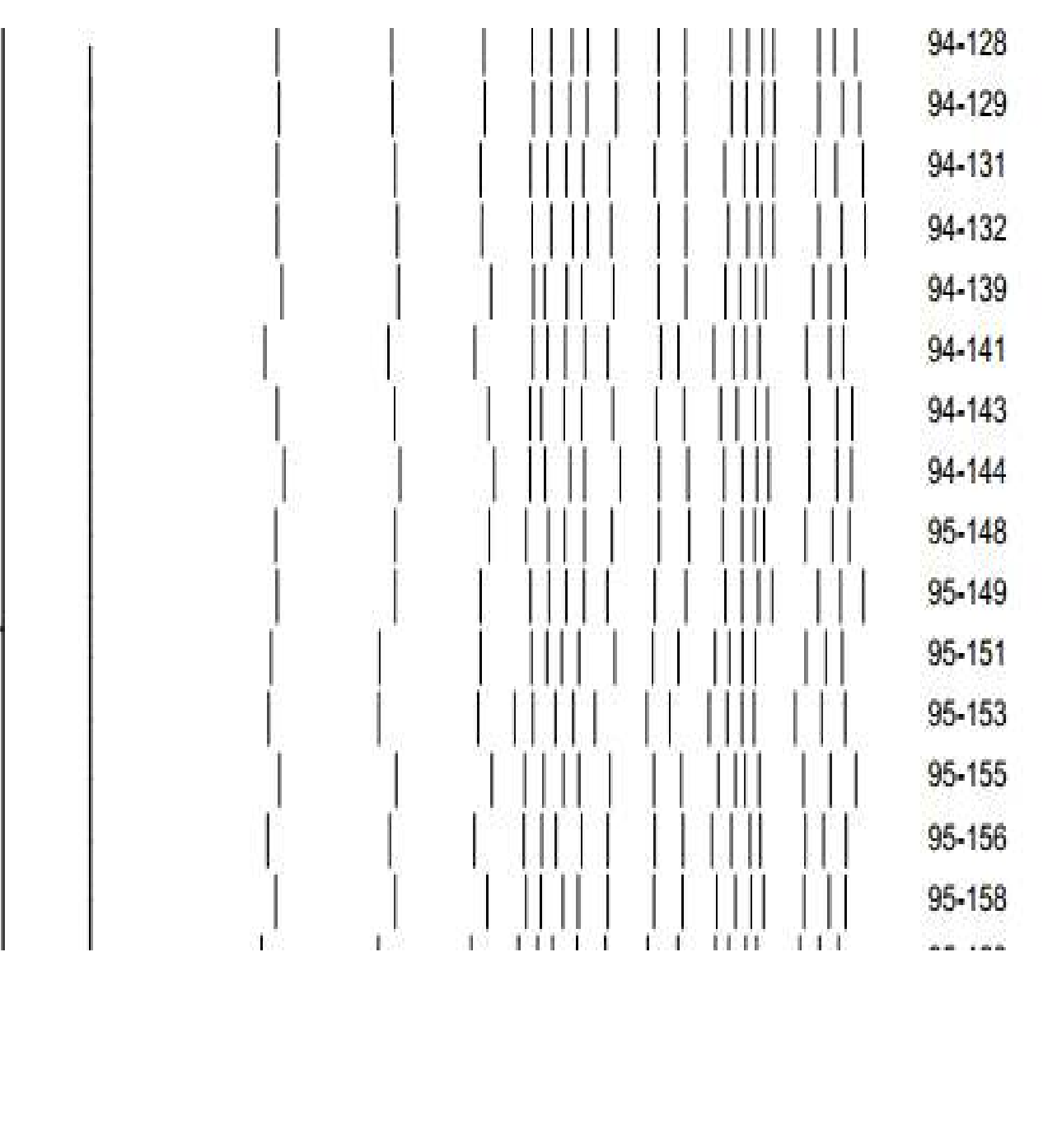